\title{
Evaluating the persistence of fluorescent and protein powders on adult blue orchard bees, Osmia lignaria (Hymenoptera: Megachilidae), for mark-capture studies
}

\author{
Natalie K. Boyle ${ }^{1}$, Scott A. Machtley ${ }^{2}$, James R. Hagler ${ }^{2}$, Theresa L. Pitts-Singer ${ }^{1}$ \\ ${ }^{1}$ Pollinating Insects Research Unit, USDA-ARS, 1410 N 800 E, Logan, UT 84341, USA \\ ${ }^{2}$ Arid-Land Agricultural Research Center, USDA-ARS, 21881 N. Cardon Lane, Maricopa, AZ 85138, USA
}

Received 19 June 2017 - Revised 20 November 2017 - Accepted 29 January 2018

\begin{abstract}
The managed, solitary bee, Osmia lignaria Say, is an efficient pollinator of orchard crops. One limitation to their commercial success is high dispersal of populations away from orchards, resulting in low establishment in provided nest cavities even when optimal pollination is achieved. While exact causes for dispersal are unknown, many existing theories remain untested due to complications with evaluating $O$. lignaria establishment in field settings. We describe relatively simple and reliable methods for passively marking $O$. lignaria with egg albumin and casein powdered proteins combined with orange fluorescent powder as they emerge from cocoons. Marked adults were examined microscopically at $0,3,6,9,12,15$, and 18 days after emergence for the fluorescent powder followed by an anti-albumin and anti-casein enzyme-linked immunosorbent assay to detect the protein powders. The orange powder and egg albumin were highly persistent on adults throughout the 18-day study, suggesting the utility of these markers for studying dispersal and retention of $O$. lignaria in the field.
\end{abstract}

\section{protein marking / ELISA / dispersal / retention / pollination}

\section{INTRODUCTION}

Osmia lignaria Say, commonly known as the blue orchard bee, is a managed solitary bee that is an effective pollinator of spring-blooming rosaceous crops such as cherry, almond, and apple (Bosch and Kemp 2000, 2001; Peterson and Artz 2014; Sheffield 2014). Native to most of the western USA and Canada (Rust 1974), this cavity-nesting bee naturally resides in wood tunnels or hollow reeds but will nest gregariously and readily in artificial nests (e.g., bundled cardboard tubes or corrugated wood blocks) when provided. $O$. lignaria are univoltine and exhibit a narrow adult foraging window spanning four to 6 weeks,

Corresponding author: N. Boyle,

Natalie.Boyle@ars.usda.gov

Marking $O$. lignaria for dispersal studies

Manuscript editor: David Tarpy which aligns comfortably with the duration of bloom in most commercially managed orchards. Its phenology can be manipulated by practicing standardized, temperature-controlled incubation protocols for overwintering cocoons. Such protocols allow for adult emergence when they are most needed for crop bloom (ranging from midFebruary to late May in most cases) (Bosch et al. 2000; Peterson and Artz 2014; Pitts-Singer et al. 2014). Due to the combined flexibility of inorchard management and timing emergence, $O$. lignaria is a compelling and promising alternative bee species for use in pollination services; this species is particularly well suited for meeting the growing pollination service demands placed on honey bees (Apis mellifera L.) for the tree fruit industry in the USA (Bosch et al. 2006).

While many details of recommended management strategies for this species are known (Bosch and Kemp 2001), current practices do not support sustainable propagation of $O$. lignaria in 
conventional environments (Artz et al. 2012, 2013). High rates of dispersal, combined with immature and adult mortality, are problematic in current efforts to domesticate $O$. lignaria for commercial applications. Additionally, low overall nest establishment and reproduction can limit orchard pollination efficacy. Causes for $O$. lignaria dispersal are unknown, particularly in environments where mass-blooming pollen and nectar resources are readily available for foraging bees. Possible explanations include factors such as method of bee release, weather, or floral resource availability (Bosch and Kemp 2001). In addition, the diameter and materials constituting $O$. lignaria nests and the distribution of nest boxes located within an orchard can influence bee dispersal and overall reproduction (Torchio 1984; Boyle and Pitts-Singer 2017). Recent work to mitigate high dispersal rates includes the development of a bee attractant that can be applied into artificial nest sites (Pitts-Singer et al. 2014) and extending the foraging season for nesting bees by planting wildflower plots near or within orchards to offer supplementary floral resources (Sheffield et al. 2008).

Until now, only limited, expensive, and timeconsuming marking methods have been available for tracking the retention and dispersal characteristics of bees such as O. lignaria . For example, emerged $O$. lignaria females can be individually marked on the dorsal side of the thorax with dots of paint and then released into an orchard for observational studies. While this technique works well for studies in enclosed greenhouses (Tepedino and Torchio 1994; Guédot et al. 2006) and small semi-field cages (Ladurner et al. 2008; Stanley et al. 2011; Artz and Pitts-Singer 2015), it is not well suited for commercial-scale field trials in which tens of thousands of bees are released per $\mathrm{km}^{2}$ to meet basic crop pollination requirements. O. lignaria retention and dispersal may also be assessed via "night counts," in which the number of bees resting inside nesting cavities after dark is counted (Monzón et al. 2004; Bosch et al. 2000; Torchio 1991). While this technique might have some utility in open field studies, it is timeconsuming and does not allow one to distinguish bees that hail from wild versus managed populations. Because $O$. lignaria are native to most of the western USA, the ability to distinguish released populations from their native counterparts is critically important in evaluating the success of proposed management protocols. Additionally, such validation would allow us to determine the relative contributions of managed bees (marked) and wild bees (unmarked) to crop pollination services.

This study was designed to develop and validate methods to mark $O$. lignaria adults for field studies of retention and dispersal. We coated yetto-emerge bee cocoons with an orange fluorescent Day-glo® powder along with egg white and bovine milk protein powders (Figure 1). We hypothesized that once adults chew through their cocoon and then crawl over their own and their neighbors' powder-coated cocoons, they would, in turn, mark themselves with the fluorescent and protein powders. Through microscopic evaluation and enzyme-linked immunosorbent assays (ELISAs), we could test whether the fluorescent and protein powder signatures on the bees were sustained over time and show that the application of powders to the cocoons of emerging bees would be a valuable technique to use in future $O$. lignaria research.

\section{MATERIALS AND METHODS}

Bee management and marking proceduresAll O. lignaria were provided by Watts Bees, Bothell, WA in the spring of 2016 as loose-cell

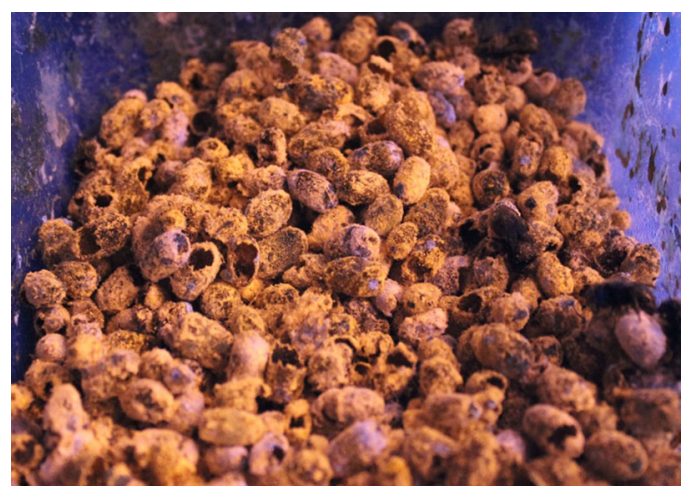

Figure 1. Loose Osmia lignaria cocoons containing mature adult bees were dusted with a combination of powdered egg albumin, milk casein, and Day-glo® fluorescent dye. Photograph taken under ultraviolet light. 
overwintering adults in cocoons. Upon receipt, bees were stored at $4{ }^{\circ} \mathrm{C}$ in darkness. On 15 May 2016, approximately 600 cocooned $O$. lignaria adults were removed from cold storage and dusted with a combination of fluorescent orange Day-glo® powder (DAYGLO Color Corp., Cleveland, $\mathrm{OH}$ ), egg white protein powder (The Barry Farm, Wapakoneta, $\mathrm{OH}$ ), and bovine milk protein powder (Sigma-Aldrich, St. Louis, MO) $(0.32,16$, and $16 \mathrm{~g}$ mixed together, respectively; Figure 1). To coat cocoons with the powder, the cocooned adults were gently stirred into the powdered mixture in a small plastic container $(22 \times 12 \times 12 \mathrm{~cm})$. The powder-marked cocoons were then stored at room temperature (approx. $22{ }^{\circ} \mathrm{C}$ ) to initiate emergence. The bees began to emerge on the same day the cocoons were removed from storage and continued to emerge until 24 May 2016.

Each emerged female was transferred to a sealed 120 -mL plastic cup. To sustain the bees in the cups, a $1-\mathrm{cm}$ high $\times 1-\mathrm{cm}$ diameter piece of flexible plastic tubing was hot glued to the center of the bottom of each cup and filled with a 1:1 sucrose solution. A 3-mm layer of sand was evenly distributed across the bottom surface around the feeder tube to provide traction for $O$. lignaria mobility.

Every day after emergence (DAE), bees were removed with forceps from their holding cups and transferred to a new cup that contained fresh sand and sucrose solution. This daily transfer was performed to eliminate secondary contact and reinoculation of the bee with any of the powders that may have contaminated the cup. After moving each bee, forceps were cleaned thoroughly with $70 \% \mathrm{EtOH}$ and Kimwipes ${ }^{\circledR}$ to prevent crosscontamination.

Female bees were sampled for persistence of the powder marks at $0,3,6,9,12,15$, and $18 \mathrm{DAE}$ from their cocoons. Thirty bees were sampled at each time interval, except for the 18-DAE treatment $(n=22)$, due to limited survival to this late stage. Each bee sampled was placed into a sterile 2-mL tube and stored at $-20^{\circ} \mathrm{C}$ for later analysis.

Detection of powdered marksAll bees were first examined microscopically (using a $\times 10$ dissecting scope) under UV light for the presence of the orange fluorescent powder. Bees were confirmed as positive for the fluorescent powder if at least one grain was detected on the body. Then, each bee was placed in a $1.5-\mathrm{mL}$ microcentrifuge tube and soaked in a $1000-\mu \mathrm{L}$ Tris-buffered saline (TBS) solution for $1 \mathrm{~h}$ at $120 \mathrm{rpm}$ on an orbital shaker. A separate $100-\mu \mathrm{L}$ aliquot of the sample solution was used for each assay to detect the egg albumin and milk casein protein by the antialbumin and anti-casein ELISAs, respectively (described by Hagler et al. 2014).

Our method of confining the bees in small containers and preventing their natural foraging and nesting activities may promote higher than expected persistence of the external markers than would be observed in their natural habitat. Therefore, a second study (and subsequent round of analyses) was conducted on our sampled bees simulating the effects of environmental pressures (e.g., heavy rainfall or exposure to in-field irrigation) to assess the persistence of the powdered markers. Specifically, all the bees were reexamined for the presence of the various powders after a succession of thorough washes. The wash treatment consisted of removing an individual bee from its original sample buffer with forceps, placing it under running tap water for $5 \mathrm{~s}$, and then rinsing it three times (10 s per rinse) with TBS. Each individual was then placed into a clean 1.5 -mL microcentrifuge tube containing $1000-$ $\mu \mathrm{L}$ TBS, soaked for $1 \mathrm{~h}$ on an orbital shaker at $120 \mathrm{rpm}$, and then re-assayed by the anti-egg albumin and anti-casein ELISA. Then each bee and the remaining sample buffer in each sample $(800-\mu \mathrm{L}$ TBS $)$ were visually re-examined for the presence of orange powder.

Negative controlsTo serve as the negative control treatment for each powder mark type, a subsample of approximately 50 untreated cocoons wereas left to emerge concurrently in a separate facility away from marked cocoons. In total, 20 negative control females were collected at emergence and stored at $-20{ }^{\circ} \mathrm{C}$. The unmarked samples were first examined visually for the presence of the orange powder and then by the ELISAs described above. A $10 \%$ false positive error rate for fluorescent powder in the negative controls prompted the initiation of a second, subsequent cohort of 
unmarked negative controls $(n=30)$ to ensure that the initial error rate was obtained as a consequence of experimenter mishandling. In this second cohort, none of the bees contained an orange powder mark.

For the ELISAs, eight negative controls were included on each 96-well ELISA plate. The protein-marked $O$. lignaria were scored positive for the presence of the respective protein marks if the ELISA optical density (OD) reading exceeded the mean negative control reading by six standard deviations (Hagler et al. 2011a).

Data analysis The percentage of bees containing the orange fluorescent powder was simply calculated for each DAE treatment regime. The positive ELISA threshold values for the bee samples were calculated as described above using unmarked, control bees. Individual bees subjected to the powdered mixture were scored positive or negative for the presence of each protein. The percentage of positive ELISA reactions was determined and the mean ( \pm s.d.) ELISA OD values were also calculated for each DAE treatment group.

\section{RESULTS}

Fluorescent powder mark retention The fluorescent orange powder was well retained on the O. lignaria females. Specifically, the orange powder was detectable on every pre-washed and postwashed bee examined, regardless of the DAE $(n=30$ for $0,3,6,9,12$, and $15 \mathrm{DAE} ; n=22$ for $18 \mathrm{DAE})$. The orange powder was most noticeable around the bee's mandibles and at the base of their wings.

Egg albumin powder mark retentionThe egg albumin protein was also well retained on the bees over the 18-day duration of the study. Specifically, the mark was detected on $100 \%$ of the pre-washed bees (Figure 2a) and on $>95 \%$ of all post-washed bees (Figure 2b). The mean OD values yielded by the egg albumin-marked bees were, in general, three to four times higher than those yielded by the milk casein-marked bees (Figure 2). The first cohort of unmarked (negative control) bees had a false positive detection rate for the presence of egg albumin on $15 \%$ (three out of 20 individuals) of the pre-washed specimens examined. However, after these bees were washed, no false positive error rate was observed. None of the second cohort of 30 negative control bees described above was positive for egg albumin powder.

Milk casein powder mark retentionThe casein mark was readily detected on 90 to $100 \%$ of the pre-washed bees for up to 9 DAE. However, the detectability of the mark steadily declined beyond the 9-DAE treatment (Figure 2c). The frequencies of casein detection on the post-washed bees were much lower (and more sporadic) than those observed for the pre-washed specimens ranging from $67 \%$ on $0 \mathrm{DAE}$ to $7 \%$ on 3 and $15 \mathrm{DAE}$ (Figure 2d). No false positives for the presence of casein were detected on either the original or second unmarked control bees.

\section{DISCUSSION}

Coating $O$. lignaria cocoons for passive selfmarking of adults was successful. Assays for both the orange colored powder and egg albumin protein powder marks revealed $100 \%$ marking efficiency with high persistence (at least $18 \mathrm{DAE}$ ) in both pre-washed and post-washed treatments. The persistence of the milk casein protein powder was less remarkable, with over $90 \%$ detection of the protein up to $9 \mathrm{DAE}$ for the pre-washed treatment. Only 7 to $67 \%$ of bees had detectable levels of casein powder once subjected to the thorough post-washed treatment, although some of the optical densities increased after the bees were evaluated. This may be due to protein powder trapped in the scopa (pollen-storing hairs located on the ventral side of a female $O$. lignaria abdomen) of the bee becoming dislodged into TBS buffer after successive washes. These results indicate that the milk casein powder would not be reliable or durable as a marker for $O$. lignaria dispersal research beyond 9 days; especially if the study area is prone to extreme weather conditions such as heavy rainfall.

It was surprising that powders from the marked bees could not be completely removed by a second washing in water or TBS buffer. It is 

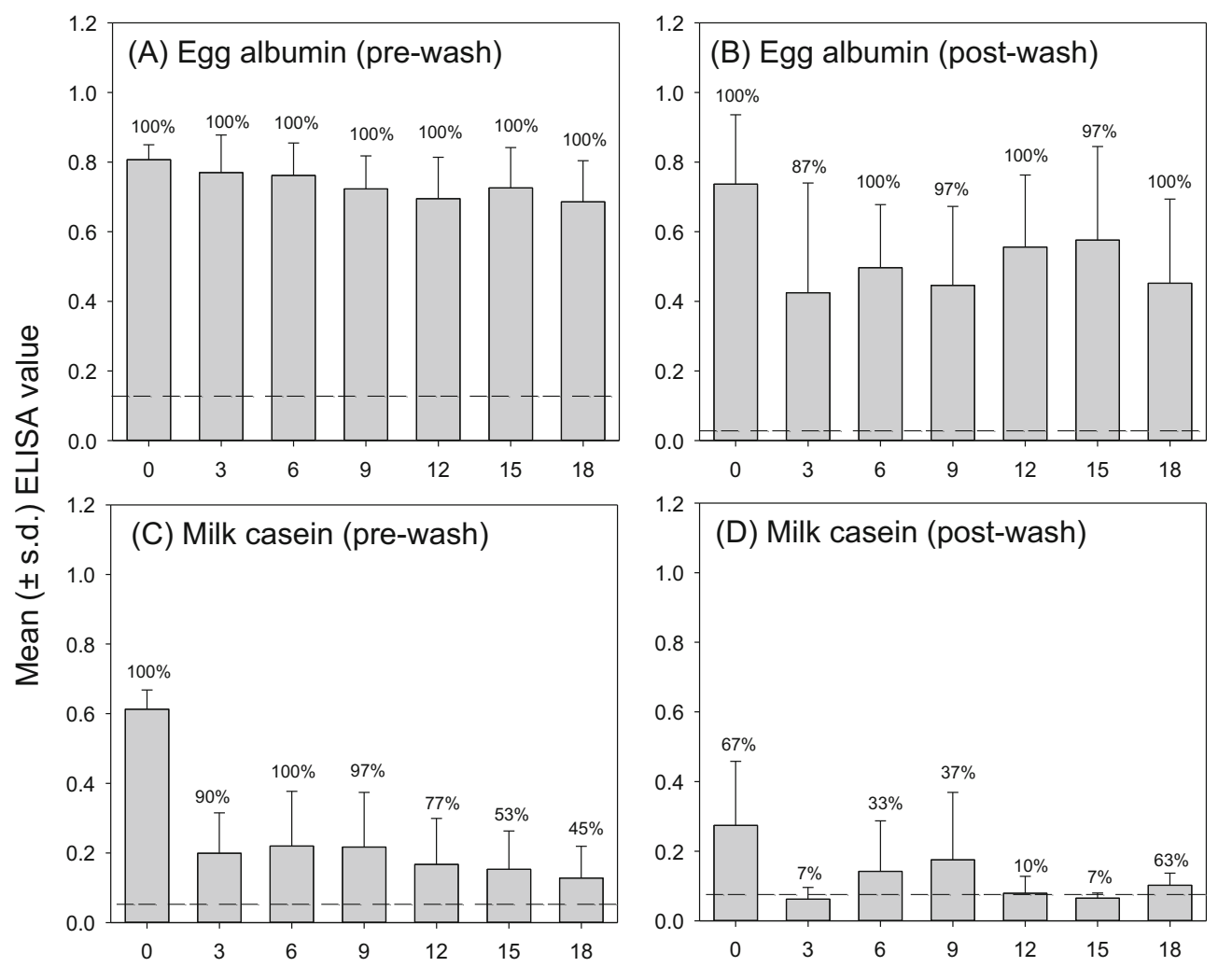

Days after emergence

Figure 2. Mean ( \pm SD) ELISA readings and percentage of Osmia lignaria specimens scoring positive (\% positive is given above the error bars of each gray vertical bar) for the presence of a egg albumin in the pre-washed specimens, b egg albumin in the post-washed specimens, $\mathbf{c}$ milk casein in the pre-washed specimens, and $\mathbf{d}$ milk casein in the post-washed specimens. Horizontal black-dashed lines represent the critical threshold values for each mark treatment ( $n=30$ individuals for each day after emergence (DAE) treatment up to $15 \mathrm{DAE} ; n=22$ for $18 \mathrm{DAE}$ ).

important to have persistent markers for O. lignaria research; females require several days after their release for emergence, mating, and locating a nest site before they commence provisioning nests and laying eggs. If environmental conditions (rain, fog, wind) and bee activity (bodily contact with nest materials and various substrates) offer no further removal of detectable markers, then fluorescent and egg albumin marking of $O$. lignaria cocoons should provide easy and reliable identification of their movement from an initial release point. Although this study extended to just $18 \mathrm{DAE}$, the high persistence of the egg albumin protein and fluorescent orange powders suggests that detectability may extend well beyond $18 \mathrm{DAE}$. This persistence suggests that the limited nesting duration of $O$. lignaria (typical range of 4-6 weeks in the spring) may not be enough time for the markers to be otherwise lost, allowing their use during nest establishment and potentially throughout the foraging period. The ELISA procedure is well suited for mass throughput. Specifically, it takes much less time than individually examining hundreds of bees for microscopic quantities of fluorescent powder under a black light (Hagler et al. 2011b). The assay is also highly sensitive and less prone to human error if minute quantities of the mark type are on the bees (JRH, unpubl. data).

Use of combining protein (egg white and casein) powders with fluorescent powders to study bee dispersal patterns is not a new concept. Egg albumin was used to mark cherry blossoms in PA, USA but was delivered to the flowers by powder-dusted 
Osmia cornifrons Radoszkowski as they escaped their emergence boxes for release in the orchards (Biddinger et al. 2013). Additionally, powdermarking was used by Hagler et al. (2011a, b) to study the area-wide dispersal of honey bees in alfalfa that was being grown for seed production. In that study, nine separate apiaries were uniquely marked with either a single fluorescent powder (e.g., orange, blue, yellow, green, or magenta) or with a combination of protein powder (e.g., egg albumin or casein) and green- and magenta-colored powder. The unique combinations of powders applied at each apiary made it easy to distinguish bees' point of origin based on the protein signature detected on their bodies. The combination of different colored powders was necessary for the apiary study, as the number of clearly distinguishable Day-glo ${ }^{\circledR}$ powders is limited. Furthermore, the study revealed no blatant evidence that the powders were transferred between bees from different honey bee colonies, as was further confirmed by Hagler et al. (2015). The practice of combining colors for eventual microscopic identification could easily carry over to applications within $O$. lignaria research activities.

Protein and fluorescent powders may be used to infer point of origin from distinct bee release sites. An important potential use of this marking technique is to address concerns over wildland trapping and interstate sales of $O$. lignaria . Current pressures on $O$. lignaria suppliers to meet commercial pollination demands lead to large-scale wild trapping across many geographically isolated locations and climates in the northwestern USA (Tepedino and Nielson 2017). Wild-trapped bees (in overwintering cocoons) are processed, stored, and distributed to native and non-native (consumer-dependent) locations. It is possible that rates of dispersal vary for managed $O$. lignaria relocated to regions that do not reflect their locally adapted range, although such studies have not yet been conducted. Our markcapture technique would be a valuable tool in evaluating differential nesting success for regionally distinct $O$. lignaria populations. Furthermore, this tool could be used to measure the effects of different release practices or release timing on managed $O$. lignaria dispersal rates.

Another example of the value of a reliable mark-capture technique is on account of
O. lignaria's native range. Because its' native range overlaps with many fruit tree production regions, immigration of wild $O$. lignaria populations into commercial orchards is not uncommon. The rate and influence of wild $O$. lignaria has not been widely studied, and their additional pollination services are likely underappreciated. However, alternative marking tools (hand-painting and counting bees at nests at night/early morning) to assess immigration of wild $O$. lignaria from managed populations are not practical or efficient for use in commercial orchards. Marking bees with egg albumin protein is relatively easy and affordable for simply distinguishing wild versus managed bees in a common landscape.

A major limitation of marking bees using the powder mixture is that it requires the destructive sampling of bees for analysis, and that removal of actively nesting female bees is not economically sensible. Individual $O$. lignaria are more valuable than honey bees, as each female is reproductive. In addition, current retail costs for $O$. lignaria are upwards of $\$ 1.00$ (USD) per female (J. Watts, pers. comm.). Such high costs are due to present challenges in propagation, trapping, and management of this species for commercial use. Furthermore, typical commercial stocking rates of O. lignaria for orchards range between 60,000 and 100,000 nesting females per $\mathrm{km}^{2}$, whereas honey bees are stocked at levels that often exceed five million females per $\mathrm{km}^{2}$ in orchards (Bosch and Kemp 2001; Sumner and Boriss 2006). Thus, the individual value and economic contribution that each female makes to orchard pollination are much greater for $O$. lignaria than are for individual honey bees. This observation highlights a need to evaluate whether protein immunomarking of $O$. lignaria can be modified to accommodate non-destructive sampling methods, such as washing live bees with TBS buffer in the orchard and immediately releasing them back into the environment.

The methods we describe will serve as valuable tools for evaluating and validating sound O. lignaria management practices aimed towards sustaining or increasing in-crop populations of managed bees. Compared to other techniques, it is cost-effective and highly sensitive and allows for monitoring of large managed bee populations 
without having to laboriously hand-paint individuals or perform exhaustive night counts.

\section{ACKNOWLEDGEMENTS}

We thank USDA ARS technicians and employees Ellen Klomps, Jenna Hanson, Matthew Treasure, Hannah Jarvis, Alan Anderson, Penina Meatoga, and Utah State University graduate students Andi Kopit and Tasha Guatney for their assistance with this experiment.

\section{AUTHOR CONTRIBUTION}

All authors contributed equally to the current manuscript. All authors read and approved the final manuscript.

\section{COMPLIANCE WITH ETHICAL STANDARDS}

Conflict of interest The authors declare that they have no potential conflict of interest in relation to the study in this paper. Mention of trade names or commercial products in this publication is solely for the purpose of providing specific information and does not imply recommendation or endorsement by the U.S. Department of Agriculture. USDA is an equal opportunity provider and employer.

Évaluation de la persistance des poudres fluorescentes et protéiques pour les abeilles adultes Osmia lignaria (Hymenoptera: Megachilidae) pour des études de capture avec marquage

marquage protéique / ELISA / dispersion / rétention / pollinisation

Untersuchung zur Persistenz fluoreszenter Proteinpulver für die Markierung adulter Mauerbienen, Osmia ligaria (Hymenoptera: Megachilidae) für Wiederfangstudien

\section{Proteinmarkierung / ELISA / Verbreitung / Persistenz / Bestäubung}

\section{REFERENCES}

Artz, D.R., Pitts-Singer, T. L. (2015) Effects of fungicide and adjuvant sprays on nesting behavior in two managed solitary bees, Osmia lignaria and Megachile rotundata. PLoS ONE. https://doi.org/10.1371/journal.pone. 0135688
Artz, D. R., Allan, M. J., Wardell, G. I., Pitts-Singer, T. L. (2012) Influence of nest box color and release sites on Osmia lignaria (Hymenoptera: Megachilidae) reproductive success in a commercial almond orchard. J. Econ. Entomol. 107 (6), 2045-2054

Artz, D. R., Allan, M. J., Wardell, G. I., Pitts-Singer, T. L. (2013) Nesting site density and distribution affects Osmia lignaria (Hymenoptera: Megachilidae) reproductive success and almond yield in a commercial orchard. Insect Conserv. Divers. 6 (6), 715-724

Biddinger, D. J., Joshi, N. K., Rajotte, E. G., Halbrendt, N. O., Pulig, C., Naithani, K. J., Vaughan, M. (2013). An immunomarking method to determine the foraging patterns of Osmia cornifrons and resulting fruit set in a cherry orchard. Apidologie 44 (6), 738-749

Bosch, J., Kemp, W. P. (2000) Development and emergence of the orchard pollinator Osmia lignaria (Hymenoptera: Megachilidae). Environ. Entomol. 29 (1), 8-13

Bosch, J., Kemp, W. P. (2001) How to manage the blue orchard bee as an orchard pollinator. Sustainable Agricultural Network, Handbook Series, Book 5, National Agricultural Library, Beltsville, MD.

Bosch, J., Kemp, W. P., Peterson, S. S. (2000) Management of Osmia lignaria (Hymenoptera: Megachilidae) populations for almond pollination: methods to advance bee emergence. Environ. Entomol. 29 (5), 874-883

Bosch, J., Kemp, W. P., Trostle, G. E. (2006) Bee population returns and cherry yields in an orchard pollinated with Osmia lignaria (Hymenoptera: Megachilidae). J. Econ. Entomol. 99, 408-413

Boyle, N. K., Pitts-Singer, T. L. (2017) The effect of nest box distribution on sustainable propagation of Osmia lignaria (Hymenoptera: Megachilidae) in commercial tart cherry orchards. J. Insect Sci. 17 (2), 41

Guédot, C., Pitts-Singer, T. L., Buckner, J.S., Bosch, J., Kemp, W. P. (2006) Olfactory cues and nest recognition in the solitary bee Osmia lignaria. Physiol. Entomol. 31, 110-119

Hagler, J. R., Mueller, S., Teuber, L. R., Van Deynze, A., Martin, J. (2011a) A method for distinctly marking honey bees, Apis mellifera, originating from multiple apiary locations. J. Insect Sci. 11 (143), 1-14

Hagler, J. R., Mueller, S., Teuber, L. R., Machtley, S. A., Van Deynze, A. (2011b) Foraging range of honey bees, Apis mellifera, in alfalfa seed production fields. J. Insect Sci. 11 (144), 1-12

Hagler, J. R., Naranjo, S. E., Machtley, S. A., Blackmer, F. (2014) Development of a standardized protein immunomarking protocol for insect mark-capture dispersal research. 2014. J. Appl. Entomol. 138 (10), 772-782

Hagler, J. R., Machtley, S. A., Blackmer, F. (2015) A potential contamination error associated with insect protein mark-capture data. Entomol. Exp. Appl. 154, 28-34.

Ladurner, E., Bosch, J., Kemp, W. P., Maini, S. (2008) Foraging and Nesting Behavior of Osmia lignaria (Hymenoptera: Megachilidae) in the Presence of Fungicides: Cage Studies. J. Econ. Entomol. 101 (3), 647653. 
Monzón, V., Bosch, J., Retana, J. (2004) Foraging behavior and pollinating effectiveness of Osmia cornuta (Hymenoptera: Megachilidae) and Apis melliera (Hymenoptera: Apidae) on "Comice" pear. Apidologie 35 (6), 575-585

Peterson, S. S., Artz, D.R. (2014) Production of solitary bees for pollination in the United States. In: MoralesRamos, J.A., Rojas, M.G., Shapiro-Ilan, D.I. (Eds), Mass production of beneficial organisms: invertebrates and entomopathogens, Elsevier Science, London pp. 653-681

Pitts-Singer, T. L., Kemp, W. P., Moreland, D., Peterson, S. S., Buckner, J. S., Hagen, M. Patent application, Docket no.: 143.12 Pitts-Singer. Bee Attractants. Filed March 7, 2014

Rust, R.W. (1974) The systematics and biology of the genus Osmia, subgenera Osmia, Chalcosmia, and Cephalosmia. Wasmann J. Biol. 32 (1), 1-93

Sheffield, C. S. (2014) Pollination, seed set and fruit quality in apple: studies with Osmia lignaria (Hymenoptera: Megachilidae) in the Annapolis Valley, Nova Scotia, Canada. J. Pollinat. Ecol. 12 (13), 120-128

Sheffield, C. S., Westby, S. M., Smith, R. F., Kevan, P. G. (2008) Potential of bigleaf lupine for building and sustaining Osmia lignaria populations for pollination of apple. Can. Entomol. 140 (5), 589-599

Stanley, C., Pitts-Singer, T.L., Bosch, J. (2011) Influence of rough handling on Osmia lignaria (Hymenoptera: Megachilidae) retention in commercial orchards. J. Econ. Entomol. 104 (3), 750-752

Sumner D. A., Boriss H. (2006) Bee-conomics and the leap in pollination fees. ARE Update Univ. Calif. Giannini Found. 3: 9-11

Tepedino, V. J., Nielson, D. (2017) Bee-rustling on the range: trap-nesting for pollinators on public lands. Nat. Area J. 37 (2), 265-269

Tepedino, V. J., Torchio, P. F. (1994) Founding and usurping: EQUALLY efficient paths to nesting success in Osmia lignaria propinqua (Hymenoptera: Megachilidae). Ann. Entomol. Soc. Am. 87 (6), 946953

Torchio, P. F. (1984) Field experiments with the pollinator species, Osmia lignaria propinqua Cresson (Hymenoptera: Megachilidae) in apple orchards: III, 1977 studies. J. Kans. Entomol. Soc. 57 (3), 517-521

Torchio, P.F. (1991) Use of Osmia lignaria propinqua (Hymenoptera: Megachilidae) as a mobile pollinator of orchard crops. Environ. Entomol. 20 (2), 590-596 